\title{
HOSPITAL AQUIRED INFECTIONS IN REHABILITATION UNIT PATIENTS: A THREE-YEAR RETROSPECTIVE STUDY.
}

KAT

\section{Vorniotakis Panagiotis $^{1}$, Anamorlidis Andreas ${ }^{1}$, Sivetidou Sofia ${ }^{1}$, Kotroni Aikaterini ${ }^{1}$, Damianakis Emmanouil ${ }^{1}$, Bakas Eleftherios ${ }^{1}$ 1Physical Medicine and Rehabilitation Department, KAT Hospital - Athens, Greece}

Introduction: Infections of resistant microorganisms in hospital patients, is a serious problem in health care institutions. Despite attempts to comply with antiseptic rules and targeted antibiotics, these infections are still a serious problem. Also increased long of stay (LoS), which is sometimes necessary, and their stay in various nursing units of the same hospital or others, contribute to the appearance of these problems.

Purpose: Demonstration of the incidence of resistant infections in long-term patients (over one month) in the physical medicine and rehabilitation clinic of KAT Hospital.

Method: We refer to the 366 patients of the previous three years (2015-2017), of which 206 were transferred to our clinic from other hospitals, while the other 160 were in-hospital transfer. The data was collected by the hospital's inpatients office, by the infection office and by the files of the Patients.

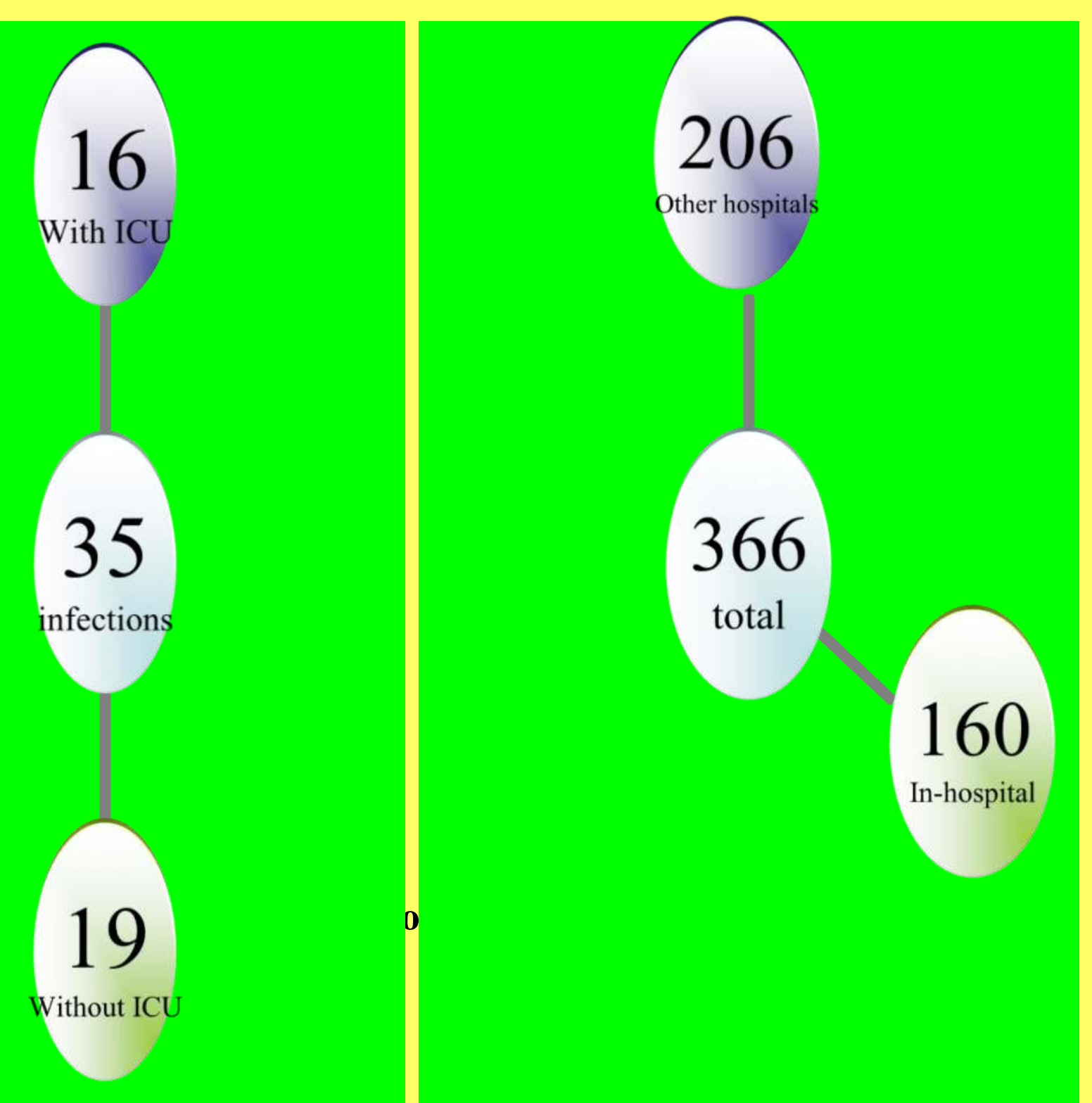

Results: Of the 366 patients hospitalized in our clinic, 35 were considered as Hospital Infections. Of these patients, 16 were hospitalized for a long time ICU of our hospital and then returned to our clinic for rehabilitation. The remaining 19 patients were been transferred to our clinic from other hospitals. In addition to the antimicrobial treatment, all had to be isolated in specific hospital chambers affecting the rehabilitation program.

Conclusion: It seems possible that, despite compliance to antisepsis rules, patients in the same hospitalisation rooms facilitates the development of hospital infections. Also the direct transfer of patients with prior hospitalization to ICU in common nursing rooms may also contribute to the transmission of resistant pathogens to the remaining patients. It is proposed that, apart from strict complience to antisepsis rules, the isolation of the patients just after their discharge from the ICU, until their microbial carrier is negative.

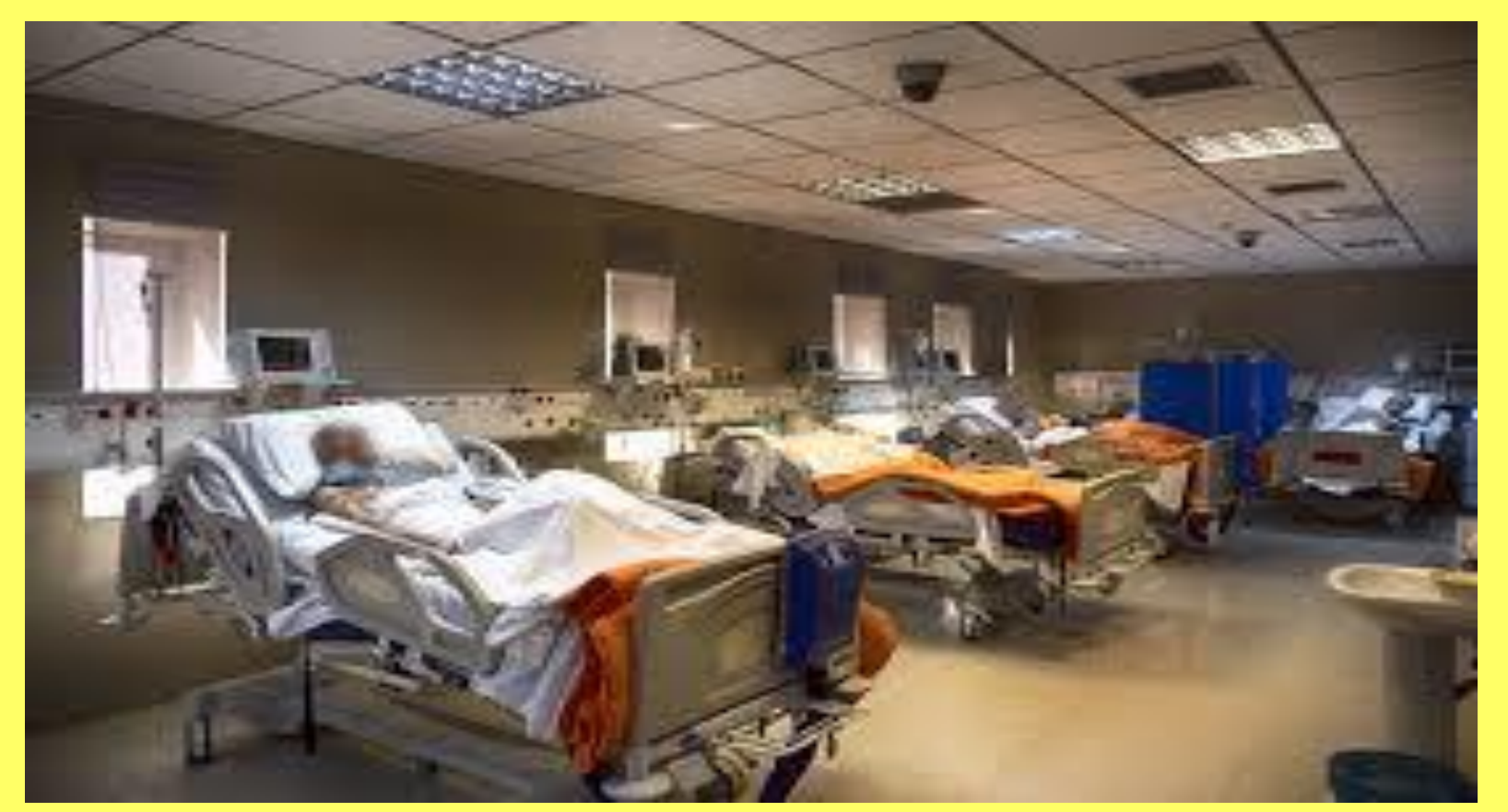

